\title{
Targeted delivery of Tet I peptide functionalized polymersomes to the rat cochlear nerve
}

This article was published in the following Dove Press journal:

International Journal of Nanomedicine

22 February 2012

Number of times this article has been viewed

\author{
Ya Zhang' \\ Weikai Zhang ${ }^{\text {* }}$ \\ Alexander $\mathrm{H}$ Johnston ${ }^{2 *}$ \\ Tracey A Newman ${ }^{3}$ \\ Ilmari Pyykkö' \\ Jing Zou' \\ 'Department of Otolaryngology, \\ University of Tampere, \\ Medical School, Tampere, Finland; \\ ${ }^{2}$ Centre for Biological Sciences, \\ ${ }^{3}$ Clinical Neurosciences, University \\ of Southampton, Southampton, UK \\ *These authors are co-contributors
}

Correspondence: Jing Zou

Department of Otolaryngology,

University of Tampere, Medical School,

B-building, Medisiinarinkatu 3, 330I4

Tampere, Finland

Tel +358401901307

Fax +358 335517700

Email jing.zou@uta.fi

\begin{abstract}
Polymersomes are nanosized vesicles formed from amphiphilic block copolymers, and have been identified as potential drug delivery vehicles to the inner ear. The aim of this study was to provide targeting to specific cells within the inner ear by functionalizing the polymersome surface with Tet1 peptide sequence. Tet1 peptide specifically binds to the trisialoganglioside clostridial toxin receptor on neurons and was expected to target the polymersomes toward the cochlear nerve. The Tet1 functionalized PEG- $b$-PCL polymersomes were administered using routine drug delivery routes: transtympanic injection and cochleostomy. Delivery via cochleostomy of Tet1 functionalized polymersomes resulted in cochlear nerve targeting; in contrast this was not seen after transtympanic injection.
\end{abstract}

Keywords: nanoparticles, peptide, round window membrane, nerve fibers, spiral ganglion cell, drug delivery

\section{Introduction}

Targeted drug delivery aims to enhance the biological availability of a chosen therapeutic agent while reducing adverse effects. The ability to target drugs to the spiral ganglion cells (SGCs) and cochlear nerve $(\mathrm{CN})$ would create an advanced therapeutic strategy for the treatment of sensorineural hearing loss. Composed of US Food and Drug Administration (FDA)-approved medical materials, poly( $\varepsilon$-caprolactone)block-poly(ethylene glycol) (PEG-b-PCL) polymersomes (PMs) are efficiently taken up by the SGCs and spiral ganglion Schwann cells. This occurs without overt toxicity in vitro spiral ganglion cultures indicating that this type of nanoparticle holds promise for drug delivery to treat sensorineural hearing loss. ${ }^{1}$ Targetability of PMs to the neural element of the cochlea was achieved through ligand mediated multivalent binding to tyrosine kinase receptors and to p75 neurotrophin receptors. ${ }^{2}$ However, no in vivo studies on the targetability of functionalized PMs were reported and there is a risk that targeting tyrosine kinase receptors and p75 neurotrophin receptors may result in altered cell signalling, which in turn may affect the neural activity and viability of the SGCs.

An alternative approach to targeting SGCs and their processes might be achieved by using the Tet1 peptide (sequence H-L-N-I-L-S-T-L-W-K-Y-R), which was identified by phage display and showed a strong affinity to differentiated PC12 cells, primary motor neurons, and dorsal root ganglion cells. Tet1 peptide binds to the trisialoganglioside clostridial toxin (GT1b) receptors, ${ }^{3}$ which are also expressed in the cochlea. ${ }^{4,5}$ Any disruption in the neural activity and viability of SGCs might be avoided because 
GT1b is not involved in SGC cell signalling. Modification of nanoparticles with the Tet1 peptide has resulted in improved targeted gene delivery to neurons both in vitro and in vivo. ${ }^{6,7}$ We hypothesized that SGCs or CN targeted drug delivery could be achieved by using PEG- $b$-PCL PMs functionalized with the Tet1 peptide.

In our previous study, unlabelled PMs that were delivered onto the round window membrane (RWM) did not appear in SGCs and spiral ganglion satellite cells. ${ }^{8}$ In the present study, PMs were functionalized with the Tet1 peptide. The targetability was evaluated using two therapeutic approaches in which the PMs were delivered via either transtympanic injection or cochleostomy. The inner ear distribution of Tet1 functionalized PMS (Tet1-PMS) was then assayed using confocal microscopy. Two different control samples of PMs, unlabelled PMs and PMs functionalized with a scrambled Tet1 sequence (ScrTet1), were used in this investigation.

\section{Materials and methods Materials}

Anhydrous $N, N$-dimethylformamide (DMF), 4-nitrophenyl iodoacetate, and dialysis tubing with a $12-14 \mathrm{kDa}$ cutoff and $6.3 \mathrm{~mm}$ diameter were purchased from Sigma-Aldrich (Gillingham, UK). Poly(ethylene glycol)-block-poly ( $\varepsilon$-caprolactone) methyl ether, PEG average $\mathrm{M}_{\mathrm{n}} \sim 5800$, PCL average $\mathrm{M}_{\mathrm{n}} \sim 19,000,\left(\mathrm{NH}_{2}\right.$-PEG5.8K- $b$-PCL19K), and $\left(\mathrm{NH}_{2}\right.$-PEG5K- $b$-PCL5K) were supplied by Polymer Source Inc, (Dorval, Canada). 1,1'-dioctadecyl-3,3,3'3'tetramethylindocarbocyanine perchlorate (DiI) was supplied by Invitrogen (Paisley, UK). Peptide sequences were synthesized by Activotec (Cambridge, UK). Phosphatebuffered saline (PBS) solution was made using PBS tablets (Oxoid, Basingstoke, UK) and $\mathrm{H}_{2} \mathrm{O}$ (purified using a Milli-Q Ultrapure Water Purification System [resistance of 18.2 $\mathrm{M} \Omega \mathrm{cm}$ at $25^{\circ} \mathrm{C}$, Livingston, Cambridge, UK). Dynamic light scattering was performed on a Zetasizer Nano Z (Malvern Instruments Ltd, Malvern, UK).

\section{Methods}

\section{Preparation of peptide functionalized polymer}

$\mathrm{NH}_{2}$-PEG5.8K- $b$-PCL19K (60 mg) was dissolved in DMF (2 mL). 4-nitrophenyl-iodoacetate $(10 \mathrm{mg})$ was added, and the reaction mixture stirred for 4 hours. Diethyl ether $(50 \mathrm{~mL})$ was added and the solution left overnight. The resulting precipitate was filtered and washed with diethyl ether to give iodoacetate-PEG5.8K- $b$-PCL19K functionalized polymer ( $45 \mathrm{mg}, 75 \%$ yield). A total of $12 \mathrm{mg}$ of the solid was dissolved into DMF $(1 \mathrm{~mL})$ to give a pale yellow solution that turned clear when cysteine terminated peptide $(1 \mathrm{mg})$ was added. The reaction mixture was stirred overnight, concentrated in vacuo, and used crude in PM preparation. The peptide sequences used were Tet1 (NH2-HLNILSTLWKYRC-COOH) and a ScrTet1 (NH2-LHNYTWLSLRKIC-COOH).

\section{PM preparation}

The carboxy cyanine dye DiI was dissolved in DMF at a concentration of $0.1 \mathrm{mg} / \mathrm{mL}$. Tet1-PEG5.8K- $b$-PCL19K $(6.0 \mathrm{mg})$ was added to the DiI/DMF solution $(0.4 \mathrm{~mL})$, and this was placed in an ultrasonic bath for 10 minutes to aid dissolution. The polymer solution was then added dropwise ( $\sim 1$ drop every 8 seconds) to rapidly stirred PBS $(1.60 \mathrm{~mL})$. The sample was then dialyzed against PBS $(400 \mathrm{~mL})$, and the buffer solution was replaced four times over the course of 48 hours, after which the sample was removed from the dialysis tubing. Control PMs with the scrambled ScrTet1 peptide (ScrTet1-PMs) and unlabelled PMs were made in an identical fashion using ScrTet1PEG5.8K- $b$-PCL19K and iodoacetate-PEG5.8K-b-PCL19K respectively (Figure 1A). Analysis by dynamic light scattering showed the typical hydrodynamic diameter of the Tet1-PMs to be $105.0 \pm 20.0 \mathrm{~nm}$. Both the ScrTet1-PMs and unlabelled PMs had hydrodynamic diameters similar to the Tet1-PMs. The zeta potential measurements for the Tet1-PMs and ScrTet1-PMs were negative, with typical measurements for the Tet1/ScrTet1-PMs and unlabelled PMs being -0.764 and $-0.578 \mathrm{mV}$, respectively. Before use in in vivo experiments, the PMs were sterile-filtered through a $0.2 \mu \mathrm{m}$ cellulose acetate syringe filter.

\section{Animals}

Twenty-seven 5-month-old male SD rats with normal Pryer's reflexes and weighing 350-450 g (supplied by the Experimental Animal Unit, University of Tampere, FL) were used in this study. All rats were treated in accordance with the directives of the regional ethics committee of the University of Tampere. All experimental procedures were performed under general anesthesia after intraperitoneal injection of a mixture of $0.8 \mathrm{mg} / \mathrm{kg}$ of medetomidine hydrochloride (Domitor, Orion, Espoo, Finland) and $80 \mathrm{mg} / \mathrm{kg}$ of ketamine hydrochloride (Ketalar; Pfizer, Helsinki, Finland).

The animals were assigned to two groups: in group 1, Tet1-PMs, ScrTet1-PMs, and unlabelled PMs were delivered by transtympanic injection bilaterally; in group 2, Tet1PMs, ScrTet1-PMs, and unlabelled PMs were delivered by cochleostomy unilaterally. Three days after administration of the PMs was begun, the animals were sacrificed and their cochleae were harvested. The cochleae were processed to either whole mounts or cryosectioning. 


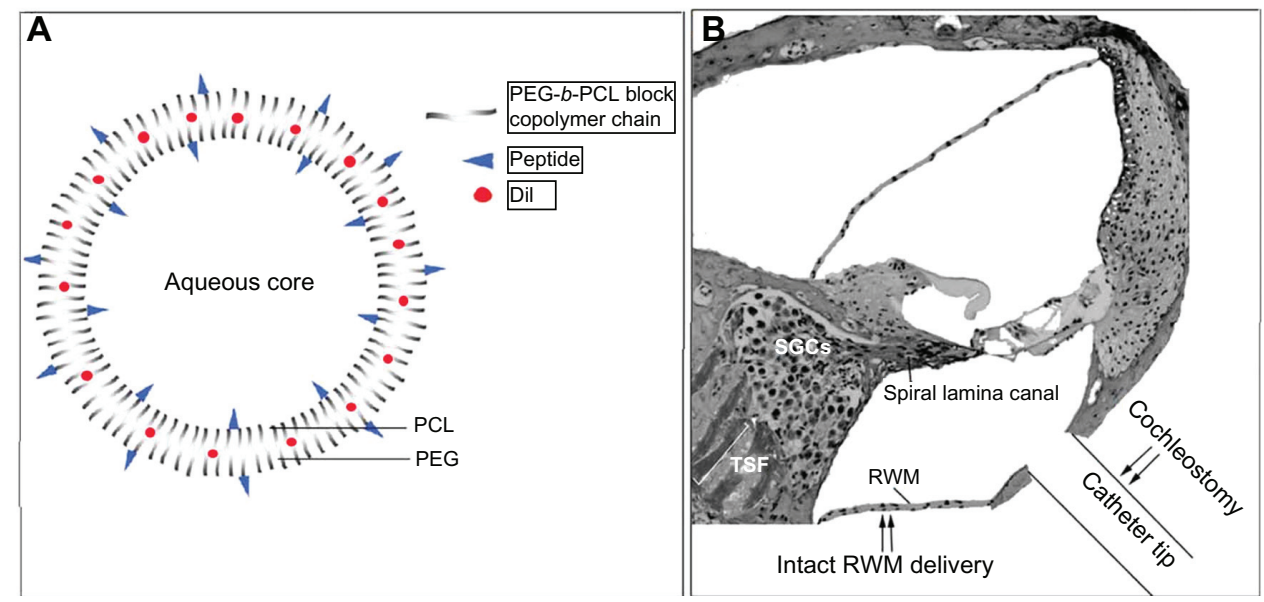

Figure I Illustration of polymersome schema and administration approaches. (A) Shows PEG-b-PCL block copolymers self-assembled in an aqueous environment to form polymersomes (PMs). The poly- $\varepsilon$-caprolactone (PCL) units of the polymer form a hydrophobic membrane; the polyethylene glycol (PEG) units form a hydrophilic corona around the interior cavity of the PM. The polymer can be functionalized with a short peptide sequence such as TAT or Tet I prior to formation. The peptide-functionalized polymer can be blended with unfunctionalized polymer and, upon formation, these peptides are present on the PM surface. (B) Shows the delivery location of the PMs via intact round window membrane (RWM) (topical RWM delivery and transtympanic injection) and cochleostomy.

Abbreviations: RWM, round window membrane; SGCs, spiral ganglion cells; TSF, tractus spiralis foraminosus.

\section{Administration of PMs to the inner ear}

Protocols for transtympanic injection and cochleostomy of PMs have previously been reported. ${ }^{8}$ Briefly, for transtympanic injection, $40 \mu \mathrm{L}$ of each PM type was injected into the middle ear cavity; for cochleostomy, an osmotic pump filled with $100 \mu \mathrm{L}$ of PMs (the PMs were diluted 1:1 with artificial perilymph) was connected to the scala tympani (ST) (via a hole in the bony labyrinth that was made with a $0.5 \mathrm{~mm}$ diameter burr) by a polyethylene tube prefilled with the same solution (Figure 1B). The analysis was carried out blind, the PMs were coded prior to administration, and evaluation of the tissue by confocal microscopy was completed before decoding.

During the procedure, the animals' eyes were protected with Terramycin eye cream (Pfizer). Atipamezole hydrochloride $(2 \mathrm{mg} / \mathrm{kg})$ was injected intraperitoneally immediately after the operation to accelerate the animals' recovery from anesthesia. Saline $(2 \mathrm{~mL})$ was administered via subcutaneous injection in the neck. Rimadyl ( $1 \mathrm{mg} / \mathrm{kg}$; Pfizer) was injected to relieve pain. Baytril (10 mg/kg; Orion, Germany) was injected intraperitoneally once a day to prevent middle ear infections.

\section{Histology analysis}

The rats were anesthetized using the procedure described above, and this was followed by fixation through cardiac perfusion with 0.01 M PBS containing $0.6 \%(\mathrm{v} / \mathrm{v})$ heparin $(\mathrm{pH} 7.4)$ and then $4 \%$ paraformaldehyde (Merck, Espoo, Finland). The isolated cochleae were rinsed with water for 1 minute to remove any PMs that may have attached to the middle ear mucosa and the round window membrane (RWM), and fixative was added for 2 hours (for whole mount samples) or overnight (for cryosection samples).
The methods for perfusion, sample preparation, and confocal microscopy after PM delivery have previously been reported. ${ }^{8}$

\section{Immunostaining}

The cryosections were incubated with 1:20 preimmunized goat serum at room temperature for 60 minutes and then incubated with one of the following primary antibodies at $4^{\circ} \mathrm{C}$ overnight: rabbit anti-neurofilament-200 (NF-200) polyclonal antibody (1:400; Sigma-Aldrich, Helsinki, Finland), rabbit polyclonal antibody to S100 (1:400; Abcam, Cambridge, UK), or rabbit polyclonal antibody to peripherin (1:100; Millipore). After washing in PBS, the specimens were incubated with fluorescein isothiocyanate-labeled goat anti-rabbit IgG (1:400; Sigma-Aldrich, USA) at room temperature for 60 minutes, followed by incubation with 4,6diamidino-2-phenylindole $(10 \mu \mathrm{g} / \mathrm{ml})$ for 10 minutes. After washing with PBS, the slides were mounted in Fluoromoun ${ }^{\mathrm{TM}}$ aqueous mounting medium (Helsinki, Finland).

\section{Fluorescence signal analysis}

Colocalization of the Tet1-PMs, ScrTet1-PMs, and unlabelled PMs with NF-200 immunostaining in confocal micrographs was analyzed using ImageJ 1.42q software (National Institutes of Health, Bethesda, MD).

\section{Results}

\section{Tet I-PMs delivered via transtympanic injection}

All the investigated PMs (Tet1-PMs, ScrTet1-PMs, and unlabelled PMs) were detected on the RWM, spiral ligament 
fibrocytes, mesothelial cells in the scala vestibuli and ST, and Reissner's membrane. However, none were present in the $\mathrm{CN}$.

\section{Tet I-PMs delivered via cochleostomy}

Distribution of PMs in the spiral ganglion

\section{and the cochlea nerve}

All of the PMs (Tet1-PMs, ScrTet1-PMs, and unlabelled PMs) were detected in the cochlea nerve (CN) within the spiral lamina canal, within the modiolus, and in the spiral ganglion satellite cells in Rosenthal's canal (Figure 2). Only the Tet1-PMs were detected in nerve fibers in the tractus spiralis foraminosus region (Figure 2 and Figure 4A), which are multiple tiny osseous canals that allow the axons to pass from the Rosenthal canal to the cochlea nerve in the modiolus.

A fluorescence signal intensity gradient was visualized from the distal to the proximal parts of the peripheral processes of the SGCs (Figure 3A-C). Nevertheless, the distribution patterns of the PMs in the peripheral processes of the SGCs were different: the Tet1-PMs were evenly distributed in the nerve fibers, while the majority of ScrTet1-PMs appeared within the epineurium surrounding the nerve bundle

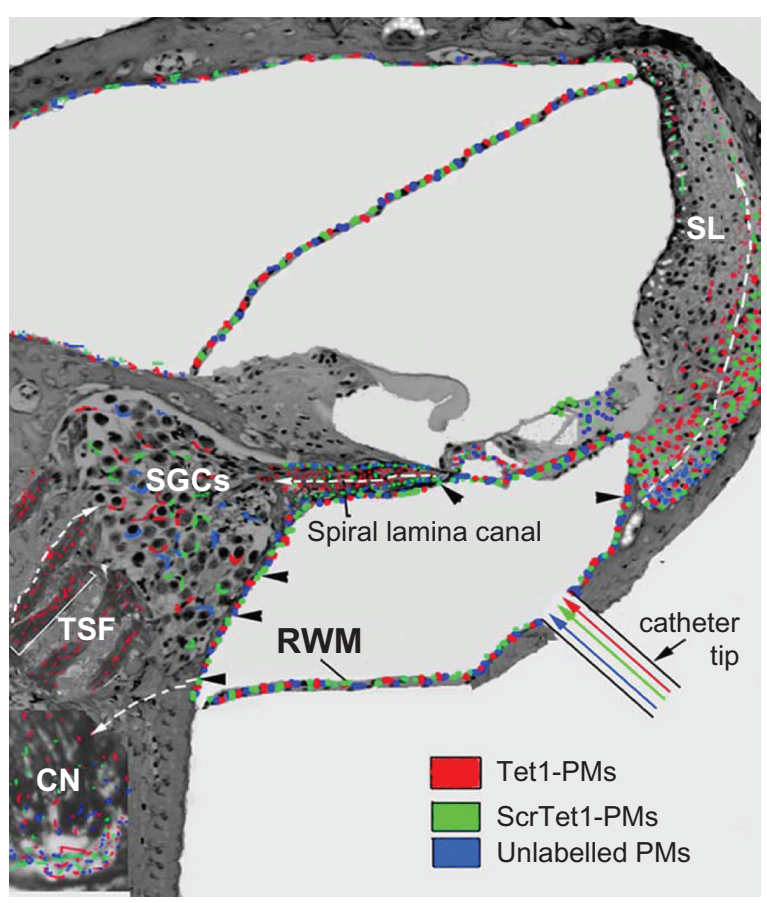

Figure 2 Illustration of the distribution of the polymersomes (PMs) after delivery using cochleostomy. In the cochlear nerve within the spiral lamina canal, the cochlear nerve in the tractus spiralis foraminosus region, and the spiral ligament (SL), the distribution patterns of Tet I-PMs, ScrTetI-PMs, and unlabelled PMs were different. Notes: Arrowheads, the openings in ST that allowed the PMs to pass through. ${ }^{19}$ White arrows with dashed lines, the possible routes of PM distribution in the inner ear. Abbreviations: TetI-PMs, TetI-functionalized PMs; ScrTetI-PMs, scrambled TetI peptide modified PMs; RWM, round window membrane; SGCs, spiral ganglion cells; $\mathrm{CN}$, cochlear nerve. (a few of the PMs were found in nerve fibers), and the unlabelled PMs were detected mainly within the epineurium with only sparse uptake in the nerve fibers of peripheral processes (Figure 2; Figure 3D-F).

The neural structures showing PM distribution were further identified by immunostaining using NF-200, S100, and peripherin. As can be seen, the Tet1-PMs were localized within or adjacent to NF-200-positive nerve fibers of SGCs (Figure 4B and F). In contrast, the scrambled and the unlabelled PMs were remote from the NF-200-positive nerve fibers (Figure 4C and D). Co-localization with S100-positive structures that represent the spiral ganglion satellite cells occurred with all PM types (Figure 5C, F, and I). None of the PMs were observed in neuronal soma of the SGCs identified by NF-200 immunostaining (Figure 5B, E, and H) and peripherin immunostaining (not shown).

\section{Distribution of PMs in RWM, cochlea scala,} and spiral ligament

All the PMs (Tet1-PMs, ScrTet1-PMs, and unlabelled PMs) were observed in the RWM, the mesothelial cells of the ST, and SV (Figure 2; Figure 3A-C), and the anterior and posterior spiral modiolar vein in the modiolus. In the scala media, PMs occasionally appeared in the inner and outer pillar cells of Corti's organ. In addition, the scrambled and the unlabelled PMs were occasionally found in Hensen cells and Claudius cells (Figure 2; Figure 3B and C), while the Tet1-PMs were not detected in these cells. Tet1-PMs and ScrTet1-PMs were detected in the entire spiral ligament. Unlabelled PMs were only detected in the type IV and V spiral ligament fibrocyte districts of the SL (Figure 2; Figure 6). ${ }^{9,10}$ In contrast, all the PMs were occasionally found in the strial vascularis.

\section{Discussion}

\section{Targeted axonal distribution of Tet I-PMs} Limited distribution of Tet I-PMs in cochlea after transtympanic injection

The Tet1-PMs administered via transtympanic injection showed no difference in distribution pattern in the cochlea compared to ScrTet1-PMs and unlabelled PMs, and no targeting to the SGCs and CN was observed. However, PMs were observed in spiral ligament fibrocytes that lack GT1b receptors, indicating non-specific uptake. Furthermore, no PMs were detected in the SGCs, a phenomenon that might be due to limited passage of the PMs through the RWM to the perilymph (Figure 2). The RWM is the major barrier between the middle ear and the cochlea, and consists in rats of three 

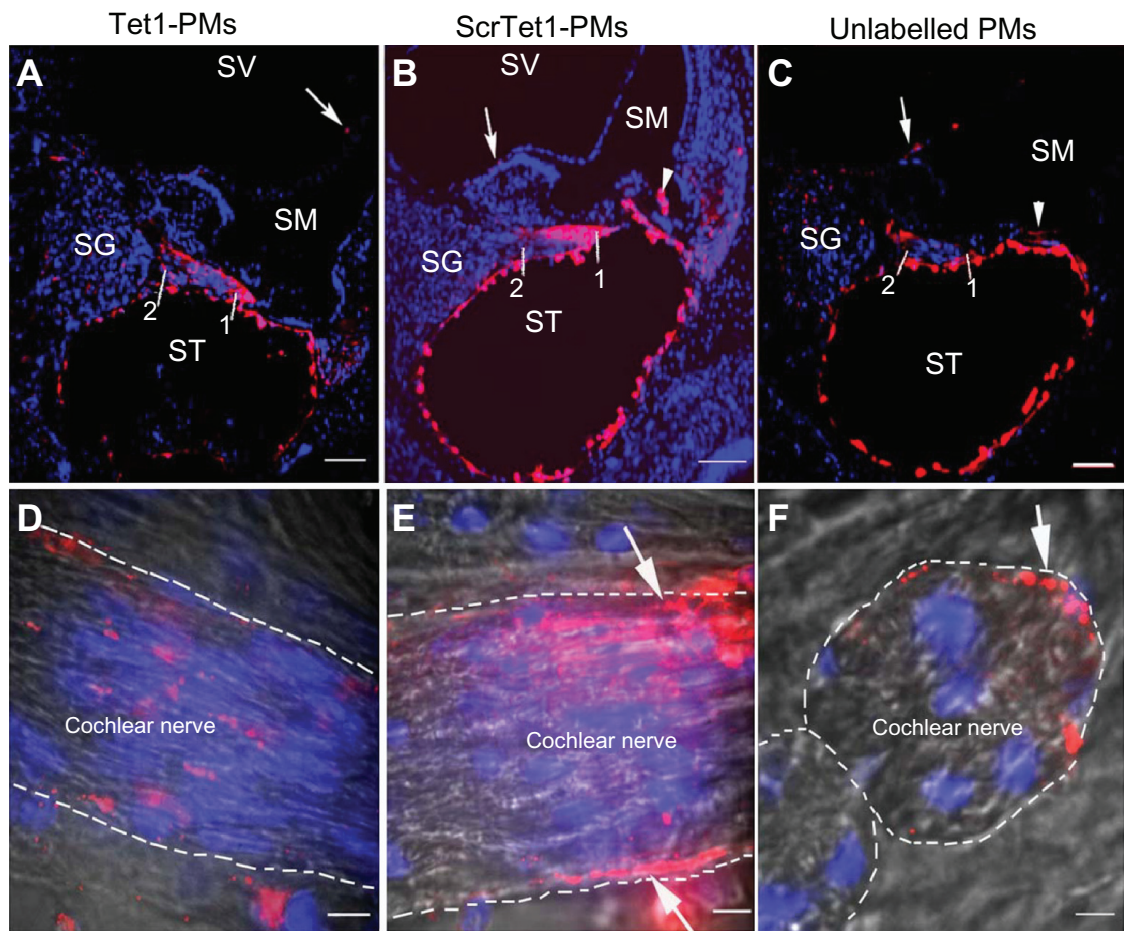

Figure 3 Distribution of TetI-PMs, ScrTetI-PMs, and unlabelled polymersomes (PMs) in rat cochlea after administration via cochleostomy. All three types of PM were detected in the mesothelial cells of ST and SV (arrows), and spiral ganglion (SG) region (A-C). The fluorescence signal intensities of Tet I-PMs and ScrTet I-PMs were higher in the distal part (point I in A and B) than that in the proximal part (point 2 in $\mathbf{A}$ and $\mathbf{B}$ ) of periphery processes of the spiral ganglion cells. Larger amount of ScrTet IPMs and unlabelled PMs were detected in the epineurium (arrows and dashed lines in $\mathbf{E}$ and $\mathbf{F}$ ) of peripheral processes than within the peripheral processes, whereas the Tet I-PMs were evenly distributed within the peripheral processes (D). $\mathbf{D}$ and $\mathbf{E}$ are longitudinal sections of peripheral processes. $\mathbf{F}$ is a transverse section of a peripheral processe.

Notes: Red: PMs. Blue: 4,6-diamidino-2-phenylindole. Gray (D-F): bright field channel. Scale bar in panels $(\mathbf{A}-\mathbf{C})=10 \mu \mathrm{m}$. Scale bar in panels $(\mathbf{D}-\mathbf{F})=100 \mu \mathrm{m}$.

Abbreviations: SV, scala vestibuli; ST, scala tympani; Tet I-PMs, Tet I-functionalized PMs; ScrTetI-PMs, scrambled Tet I peptide modified PMs.
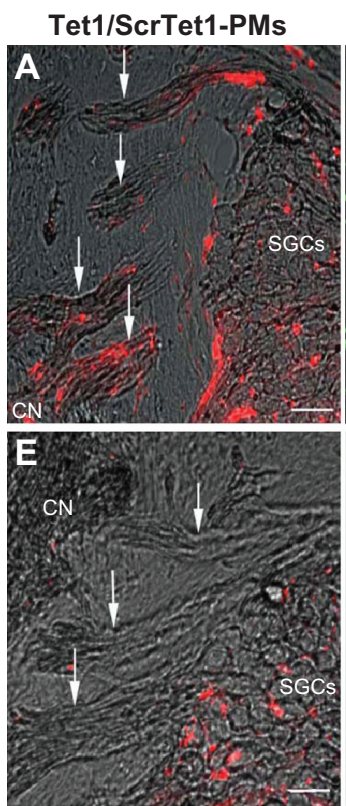

\begin{abstract}
Tet1-PMs
\end{abstract}

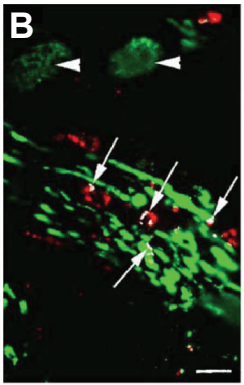

ScrTet1-PMs

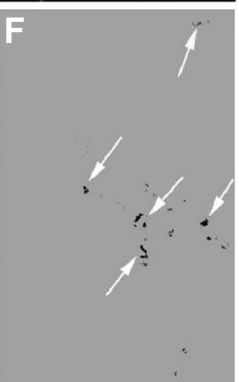

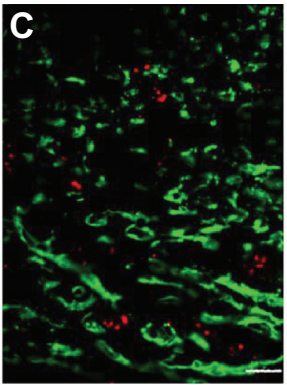

Unlabelled PMs
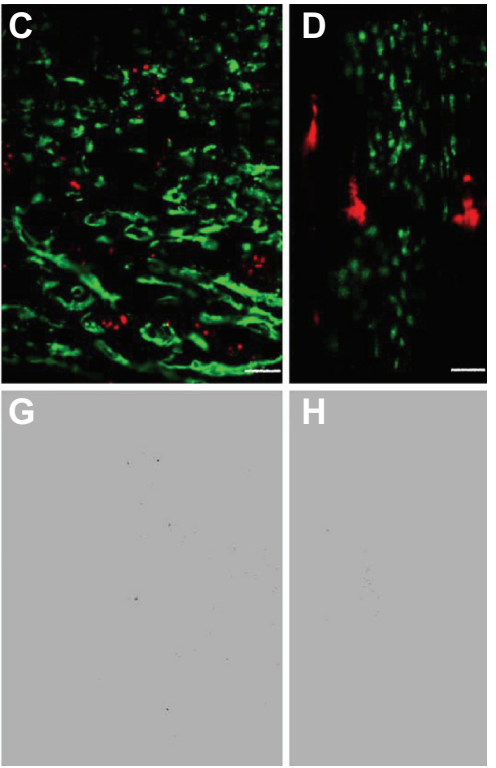

Figure 4 Location of TetI-PMs, ScrTetI-PMs, and unlabelled polymersomes (PMs) in the cochlear nerve after administration via cochleostomy. Tet I-PMs (A) and ScrTet IPMs (E) were detected in the spiral ganglion (SG) and the nerve fibers in the tractus spiralis foraminosus region of the CN. Only Tet I-PMs were detected in the tractus spiralis foraminosus region (arrows in A). Panels B-D show the co-localization of the PMs with NF-200 (arrows in panel B). The co-localized points are indicated by black dots in panels $\mathbf{F}$ (arrows), $\mathbf{G}$ and $\mathbf{H}$ (analyzed by Imagej software). Arrowheads in panel $\mathbf{B}$ pointed to SGCs.

Notes: Red: PMs. Green: NF-200 immunostaining. Blue: 4,6-diamidino-2-phenylindole. Gray: bright field. Scale bar in $\mathbf{A}$ and $\mathbf{E}=25 \mu \mathrm{m}$; scale bar in B-D = $10 \mu \mathrm{m}$. Abbreviations: CN, cochlear nerve; NF-200, neurofilament 200; TetI-PMs, Tet I-functionalized PMs; ScrTetI-PMs, scrambled Tet I peptide modified PMs. 

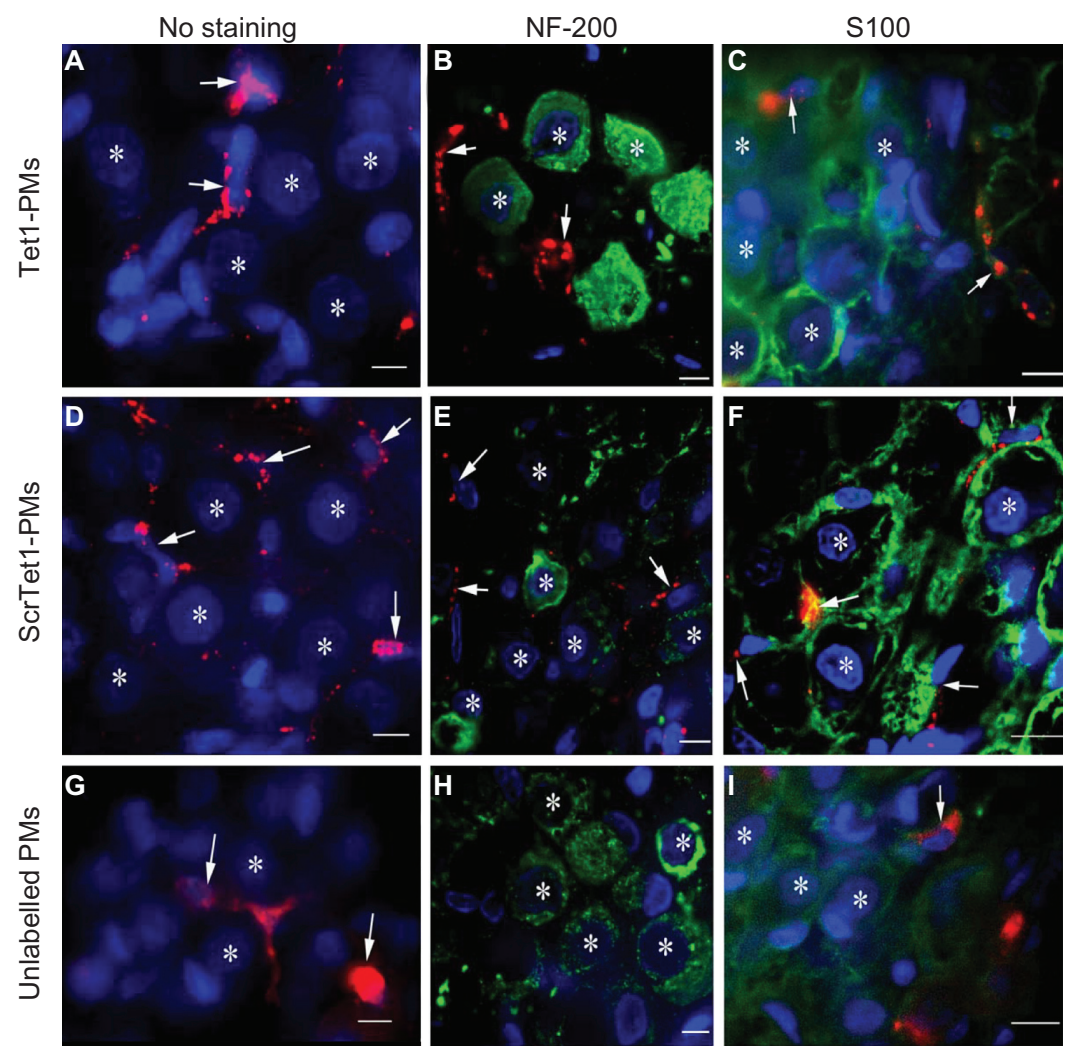

Figure 5 Distribution of Tet I-PMs, ScrTetI-PMs, and unlabelled polymersomes (PMs) in the spiral ganglion architectures after administration via cochleostomy. All three types of PM administrated by cochleostomy were located in the spiral ganglion satellite cells (SGSCs, arrows). PMs were not detected in the neuronal soma of the spiral ganglion cells. Notes: *Nucleus of type I spiral ganglion cells. Red: PMs. Green: NF-200 or SI00 immunostaining. Blue: 4,6-diamidino-2-phenylindole. Scale bar = I0 $\mu$ m.

Abbreviations: TetI-PMs, Tetl-functionalized PMs; ScrTetI-PMs, scrambled Tetl peptide modified PMs.

layers: an outer epithelial layer that has tight junctions on the middle ear side and a continuous basement membrane beneath, a connective tissue core, and an inner endothelial layer. ${ }^{11}$ Permeability of the RWM is poor, and only $0.01 \%-0.0001 \%$ of the original concentration of proteins applied on the RWM was detected in the inner ear perilymph. ${ }^{12,13}$ This is supported by our observation that PMs were present in the SGCs when administrated via cochleostomy, in which
PMs are directly administered to the perilymph, avoiding the RWM.

\section{Neurofilament targeting of the Tetl-PMs after intracochlear administration}

After delivery via cochleostomy, Tet1-PMs demonstrated axonal targeting, as identified by the localization of Tet1-PMs within or adjacent to neurofilaments, and their migration to the
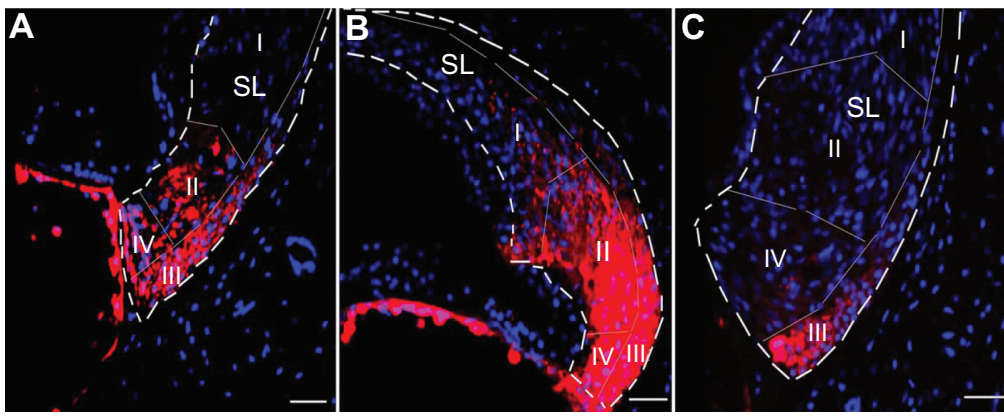

Figure 6 Distribution of polymersomes with and without Tet I functionalization in the spiral ligament after administration via cochleostomy. A shows the Tet I-PMs detected in type II, III, and IV spiral ligament (SL) fibrocyte district. B shows the ScrTet I-PMs detected in type I, II, III, and IV spiral ligament fibrocyte district. C shows the unlabelled PMs in type III spiral ligament fibrocyte district.

Notes: Red: PMs. Blue: 4,6-diamidino-2-phenylindole. Scale bar $=50 \mu \mathrm{m}$

Abbreviations: Tetl-PMs, Tetl-functionalized PMs; ScrTetI-PMs, scrambled Tetl peptide modified PMs. 
tractus spiralis foraminosus region. Tet1 peptide is reported to bind to the GT1b receptor, which exists abundantly in neuronal membranes in the central nervous system ${ }^{14}$ and peripheral nerve axolemma. ${ }^{15}$ All the PMs (whether with or without the Tet1 peptide) were observed in spiral ganglion satellite cells of the spiral ganglion and the $\mathrm{CN}$ in the modiolus, as identified by S100 immunostaining, but only the Tet1-PMs were detected in nerve fibers identified by NF-200 immunostaining.

Neurons have active axonal transport machinery that moves substances from their distal terminals to the neuronal soma. ${ }^{16}$ We hypothesized that the distribution of Tet1-PMs in the tractus spiralis foraminosus region (Figure 4A) was a consequence of active axonal transport from the $\mathrm{CN}$ in the modiolus to the neural soma of SGCs. However, the Tet1-PMs were not observed in the neuronal soma. This may be because the SGCs are enclosed by spiral ganglion satellite cells and there is no communication between these cells - the Tet1-PMs were located in distal axon compartments of SGCs that have little or no communication with the cell body compartment. ${ }^{17,18}$ In addition, gene expression in the lateral ventricle was reported after administration of Tet1-PEG- $b$-polyethylenimine containing plasmid DNA. ${ }^{6}$ This indicated that the PMs might release their cargos in the distal axon compartment, which could then penetrate separately into the cell body compartment of the neuron. Thus, Tet1-PMs could target the neurofilament of the $\mathrm{CN}$; however, whether they effectively release their cargo into the cytoplasm needs further investigation.

\section{Affinity of PMs to spiral ganglion satellite cells and spiral ligament fibrocytes}

The Tet1-PMs, ScrTet1-PMs, and unlabelled PMs administrated via cochleostomy were detected in spiral ganglion satellite cells. This suggested nonspecific affinity between these PMs and the spiral ganglion satellite cells. This is in line with previous in vitro studies with cochlear explants $^{2}$ and spiral ganglion mixed cultures. ${ }^{1}$

We suggest that the reason more Tet1-PMs and ScrTet1PMs than unlabelled PMs were detected in the SL may be because the surface modification of PMs using the Tet1 and ScrTet1 peptides changes their affinity with spiral ligament fibrocytes. The PMs observed in spiral ligament fibrocytes were there due to non-specific uptake because the spiral ligament fibrocytes lack GT1b receptors.

\section{Potential routes of PM distribution in the inner ear}

After entry into the perilymph within the scala tympani, the PMs may migrate to the modiolus via openings in the modiolar wall of the ST and SV. They could then enter the peripheral processes of SGCs, either through openings in the modiolar wall near the osseous spiral lamina or through the "tunnel fibers" in the Corti's organ, and follow the CN from its distal to proximal part. ${ }^{19,20}$ This proposal is supported by the observation that the PM signal in the peripheral processes of the SGCs appears as a gradient (Figure 3). The PMs could then distribute in the lateral wall through the mesothelial sheet of the SL facing the ST (Figure 2). ${ }^{19,20}$ Thereafter, PMs may migrate within the modiolus and lateral wall and reach the higher turns of the cochlea. ${ }^{21}$ PMs from the lateral wall were detected in the Hensen cells and Claudius cells so may also contribute to the distribution seen in the Corti's organ. This result was comparable to the intracochlear distribution of lipid core nanocapsules, which are PMs of a similar size. ${ }^{20}$

\section{Conclusion}

The Tet1-PMs targeted axons of the SGCs after intracochlear administration, and it was suggested that the Tet1PMs targeted axons through GT1b receptors. However, the Tet1-PMs were not detected in the neuronal soma of the SGCs. Tet1-PMs, ScrTet1-PMs, and unlabelled PMs were detected non-specifically in SGSCs and SL. The specific targeting of Tet1-PMs to axons could allow them to be used for drug delivery to the peripheral and central nervous systems in the treatment of hearing loss and other neurological diseases.

\section{Acknowledgments}

This study was supported by the European Community 6th Framework Programme on Research, Technological Development and Demonstration (Nanotechnologybased Targeted Drug Delivery. Contract number: NMP4-CT-2006-026556, Project acronym: NANOEAR).

\section{Disclosure}

The authors report no conflicts of interest in this work.

\section{References}

1. Anderson M, Johnston AH, Newman TA, Dalton PD, RaskAndersen $\mathrm{H}$. Internalization of nanoparticles into spiral ganglion cells. J Nanoneuroscience. 2008;1:1-10.

2. Roy S, Johnston AH, Newman TA, et al. Cell-specific targeting in the mouse inner ear using nanoparticles conjugated with a neurotrophinderived peptide ligand: potential tool for drug delivery. Int J Pharm. 2010; 390:214-224.

3. Liu JK, Teng Q, Garrity-Moses M, et al. A novel peptide defined through phage display for therapeutic protein and vector neuronal targeting. Neurobiol Dis. 2005;19:407-418.

4. Maguchi S, Gasa S, Matsushima J, Saga Y, Kawano M, Makita A. Glycolipids in rat cochlea. Auris Nasus Larynx. 1991;18:1-8. 
5. Santi PA, Mancini P, Barnes C. Identification and localization of the GM1 ganglioside in the cochlea using thin-layer chromatography and cholera toxin. J Histochem Cytochem. 1994;42:705-716.

6. Kwon EJ, Lasiene J, Jacobson BE, Park IK, Horner PJ, Pun SH. Targeted nonviral delivery vehicles to neural progenitor cells in the mouse subventricular zone. Biomaterials. 2010;31:2417-2424.

7. Park IK, Lasiene J, Chou SH, Horner PJ, Pun SH. Neuron-specific delivery of nucleic acids mediated by Tet1-modified poly(ethylenimine). J Gene Med. 2007;9:691-702.

8. Zhang Y, Zhang W, Johnston AH, Newman TA, Pyykko I, Zou J. Improving the visualization of fluorescently tagged nanoparticles and fluorophore-labeled molecular probes by treatment with $\mathrm{CuSO}_{4}$ to quench autofluorescence in the rat inner ear. Hear Res. 2010;269:1-11.

9. Mahendrasingam S, Bebb C, Shepard E, Furness DN. Subcellular distribution and relative expression of fibrocyte markers in the $\mathrm{CD} / 1$ mouse cochlea assessed by semiquantitative immunogold electron microscopy. J Histochem Cytochem. 2011;59:989-1000.

10. Trowe MO, Maier H, Schweizer H, Kispert A. Deafness in mice lacking the T-box transcription factor Tbx18 in otic fibrocytes. Development. 2008;135:1725-1734.

11. Goycoolea MV, Lundman L. Round window membrane. Structure function and permeability: a review. Microsc Res Tech. 1997;36:201-211.

12. Endo T, Nakagawa T, Kita T, et al. Novel strategy for treatment of inner ears using a biodegradable gel. Laryngoscope. 2005;115:2016-2020.

13. Lundman L, Juhn SK, Bagger-Sjoback D, Svanborg C. Permeability of the normal round window membrane to Haemophilus influenzae type b endotoxin. Acta Otolaryngol. 1992;112:524-529.
14. Ryu JK, Shin WH, Kim J, et al. Trisialoganglioside GT1b induces in vivo degeneration of nigral dopaminergic neurons: role of microglia. Glia. 2002;38:15-23.

15. Sheikh KA, Deerinck TJ, Ellisman MH, Griffin JW. The distribution of ganglioside-like moieties in peripheral nerves. Brain. 1999;122: 449-460.

16. Lalli G, Schiavo G. Analysis of retrograde transport in motor neurons reveals common endocytic carriers for tetanus toxin and neurotrophin receptor p75NTR. J Cell Biol. 2002;156:233-239.

17. Vance JE, Pan D, Vance DE, Campenot RB. Biosynthesis of membrane lipids in rat axons. $J$ Cell Biol. 1991;115:1061-1068.

18. Cui B, Wu C, Chen L, et al. One at a time, live tracking of NGF axonal transport using quantum dots. Proc Natl Acad Sci U S A. 2007;104: 13666-13671.

19. Rask-Andersen H, Schrott-Fischer A, Pfaller K, Glueckert R. Perilymph/ modiolar communication routes in the human cochlea. Ear Hear. 2006;27:457-465

20. Zou J, Saulnier P, Perrier T, et al. Distribution of lipid nanocapsules in different cochlear cell populations after round window membrane permeation. J Biomed Mater Res B Appl Biomater. 2008;87:10-18.

21. Salt AN, Ma Y. Quantification of solute entry into cochlear perilymph through the round window membrane. Hear Res. 2001;154:88-97.
International Journal of Nanomedicine

\section{Publish your work in this journal}

The International Journal of Nanomedicine is an international, peerreviewed journal focusing on the application of nanotechnology in diagnostics, therapeutics, and drug delivery systems throughout the biomedical field. This journal is indexed on PubMed Central, MedLine, CAS, SciSearch $\AA$, Current Contents ${ }^{\circledR} /$ Clinical Medicine,

\section{Dovepress}

Journal Citation Reports/Science Edition, EMBase, Scopus and the Elsevier Bibliographic databases. The manuscript management system is completely online and includes a very quick and fair peer-review system, which is all easy to use. Visit http://www.dovepress.com/ testimonials.php to read real quotes from published authors. 